\title{
Advances in light-in-flight HPIV for the study of wind tunnel flows
}

\author{
S.F. Herrmann and K.D. Hinsch \\ Applied Optics Group, Carl von Ossietzky University, P.O. Box 2503, \\ D-26111 Oldenburg, Germany
}

\begin{abstract}
The concept of light-in-flight holographic particle image velocimetry (LiF-HPIV) has been demonstrated successfully for ruby laser light illumination and virtual-image reconstruction. To circumvent the many disadvantages of ruby lasers the method was transferred to illumination with pulsed second-harmonic Nd:YAG-laser light. Furthermore, real-image interrogation was employed to minimize alignment and calibration problems. By using cw Nd:YAG lasers it is possible to design an optical setup for the reconstruction that is separate from the recording equipment. Thus, the time-consuming interrogations no longer block the flow measurements.
\end{abstract}

\section{Introduction}

Up to now, the investigation of highly complex flows, as in turbulence phenomena, relies on non-invasive point-wise measuring techniques (onedimensional, 1D) or planar imaging methods (two-dimensional, 2D). Both yield up to three components (3C) of the velocity by evaluating information from light scattered by small tracer particles in the flow. As of today, fully three-dimensional (3D3C) techniques, however, are hardly used due to their complexity. Instead, such problems are treated by methods of computational fluid dynamics (CFD) because the recent increase in computing power has allowed to treat even complex geometries with larger numbers of grid points. Nevertheless, our physical understanding of complex flow phenomena is still incomplete, and numerical methods need supporting measurements for validation. 
Holographic particle image velocimetry (HPIV) - as a truly instantaneous full-field method - has recently gained increasing attention (Hinsch 2002). Meanwhile it has been used even as a validation-tool for numerical simulations (Tao et al. 2000) as well as for investigations of quasi timeseries by phase-locked measurements ( $\mathrm{Pu}$ et al. 2002). HPIV is advancing to become a versatile and feasible technique - still with huge demands on computing power and technical equipment, yet.

Among the current problems of this technique - aside from the complexity in the optical setup already mentioned - are disturbances by noise sources which are intrinsic to the physical imaging process and the still cumbersome use of photographic recording materials that are inevitable to obtain high-resolution images from dense particle ensembles over a wide and deep flow field. For the latter problem more user-friendly recording materials that avoid chemical processing and allow for in-situ development are investigated. First efforts are even made to turn particle holography completely electronic and digital. Presently, however, these techniques are still inferior to photographic film by their limited dynamic range, resolution and size and are thus only of marginal importance for highly sophisticated flow recordings. Concerning the noise problem, also optical solutions are under investigation. A recent approach utilizing the properties of a light wave with short-coherence will be used in the present setup.

Air-flows, and especially those of high complexity, are yet difficult to investigate with HPIV since $\mu \mathrm{m}$-size particles have to be introduced to faithfully follow the flow. Furthermore, a volume of typically several centimeters in size in all dimensions has to be recorded simultaneously. Finally, the relevant flow structures are often in the sub-millimeter range and require dense seeding to sample with sufficient accuracy. To preserve a given signal-to-noise ratio, however, the number density $n_{s}$ of the seeding particles must not exceed a maximum value which grows with the imaging aperture and decreases with the depth of the recorded volume (Pu et al. 2002). Thus, there are two possibilities to properly record such flows:

(1) The aperture may be increased which, however, usually goes with an increasing complexity of the setup (Zhang et al. 1997) or confronts with a variety of unwanted effects related to the recording materials (e.g. emulsion shrinkage and mechanical stability of the substrate) and the optics involved (Barnhart et al. 1994). Such setups are thus unsuitable for wind tunnel measurements, were industrial needs require practically manageable equipment. 
(2) On the other hand, the effective depth must be reduced which can be achieved even without decreasing the overall depth of the measurement volume by utilizing coherence requirements of holography. An increase in effective signal-to-noise ratio or, accordingly, a higher maximum number density of particles are possible in a setup which has been called light-in-flight holography in other context ( $\mathrm{LiFH})$ (Abramson 1996)

For applications in flow investigations this method needs a light source with a coherence length of only a few millimeters. Holography relies on the interference of object and reference wave at the position of the photographic plate. The resulting intensity modulation is recorded, serves as a diffraction grating during reconstruction and causes light from the reference beam to rebuild the object wave. Obviously, scattered light from particles and the reference wave can interfere only when their path difference is less than the coherence length of the light. As shown in the example of figure 1, reference light incident from the left has to travel a longer path to the right side of the holographic plate than to the left. Object light scattered from particles is recorded only if its path-length differs by no more than the coherence length $\mathrm{L}$ from that of the corresponding reference light. Thus, with proper alignment, particles from a shell in the middle of the observed field are recorded in a small region in the middle of the plate, particles from a front shell on the left and from a rear shell on the right. Upon reconstruction through a small aperture only a shell with a depth of roughly half a coherence length shows up.
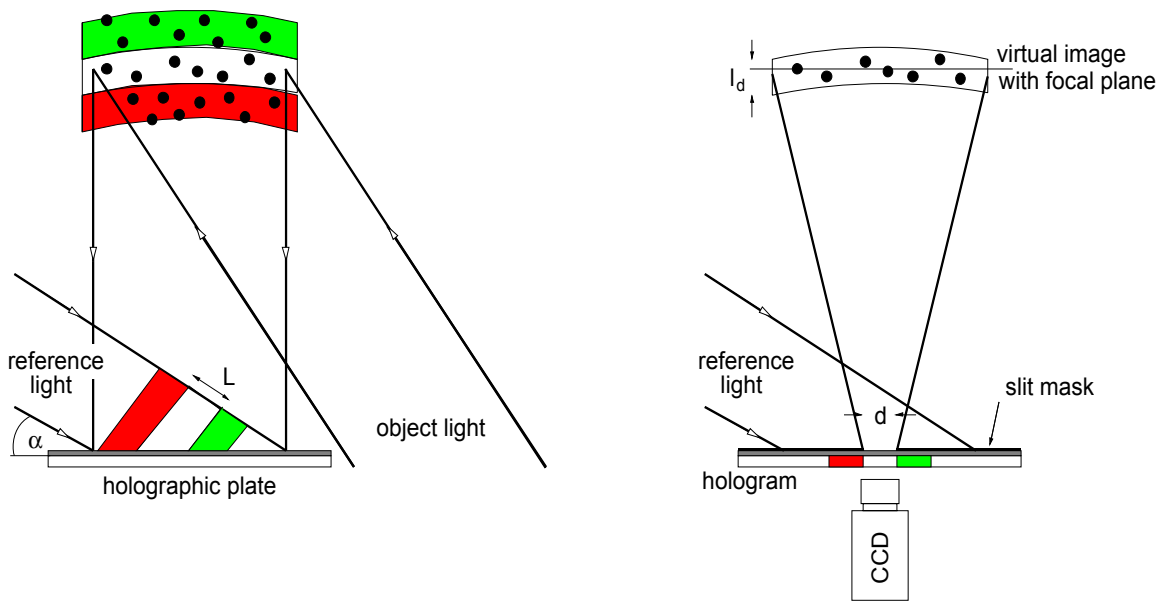

Fig. 1. Schematic of light-in-flight holography with short coherence length for particle recording (left) and reconstruction of particle images (right). 
In first experiments of light-in-flight holography for quantitative flow velocimetry (LiFH-PIV) a ruby laser was modified for short coherence (Hinrichs et al. 1997). The method has been demonstrated successfully in a study of irregular vortex shedding behind a cylinder in a wind tunnel using water-glycerin particles of a few $\mu \mathrm{m}$ in diameter (Hinrichs et al. 1998). The evaluation of the double-exposure light-in-flight holograms was still carried out by auto-correlation analysis which basically restricts the dynamic range of the system and requires a minimum particle displacement to ensure correct measurements.

The present study is dedicated to several improvements of this method. First of all, the ruby laser setup has been modified by electro-optic reference beam switching to allow cross-correlation evaluation. For long-range future work, however, a more convenient laser source is required offering a higher repetition rate to simplify alignment work, providing the necessary coherence properties and allowing almost arbitrary pulse separations. A high-energy pulsed Nd:YAG system was designed for this purpose and various components of the experiment were adapted to the green wavelength. Furthermore, the evaluation of particle images had to be done in the real image which called for a reproducible complex-conjugate reconstructing wave and a scanning and image storing device in three dimensions. Finally, a complete setup for light-in-flight holographic velocimetry (LiF-HPIV) was then tested at a wind-tunnel facility.

\section{Double-pulse ruby laser: cross-correlation evaluation}

To enable an evaluation in HPIV by cross-correlation it is necessary to record two holograms in succession which can be reconstructed separately. The holograms can be separated either spatially (e.g. on different areas of the same plate or on different plates) or, in the case of superimposed recordings on one plate, by using reference waves incident at different angles. The latter - often referred to as angular multiplexing - is widely used in HPIV measurement systems and has also been applied in the present case. In LiFH, however, there occurs a special problem, because the angle of the obliquely incident reference beam determines which region in depth of the virtual image is visible from a certain location on the hologram. Each slit aperture normal to the plane of incidence reproduces a sheet in depth. Furthermore, to avoid misalignments between the images from first and second recording it is necessary to reconstruct the same depth from both holographic images without changing the position of the CCD camera, i.e. through the same aperture. As a consequence the propagation di- 
rection of the reference waves had to be chosen in such a way that they are incident on the plate with the same azimuthal angle but differ in the vertical angle - in the present case by about $10^{\circ}$. Cross-talk between the images is thus prevented sufficiently. Such an arrangement is depicted in figure 2.

For the recording of the holograms a double-pulse ruby laser ( $\lambda=694 \mathrm{~nm}, 5 \mathrm{~J}$ per pulse) has been used. The separation of pulses for the two reference beams was produced by a Pockels cell followed by a polarizing beam-splitter. The electro-optic effect is used to rotate the polarization of the second laser pulse by $90^{\circ}$.

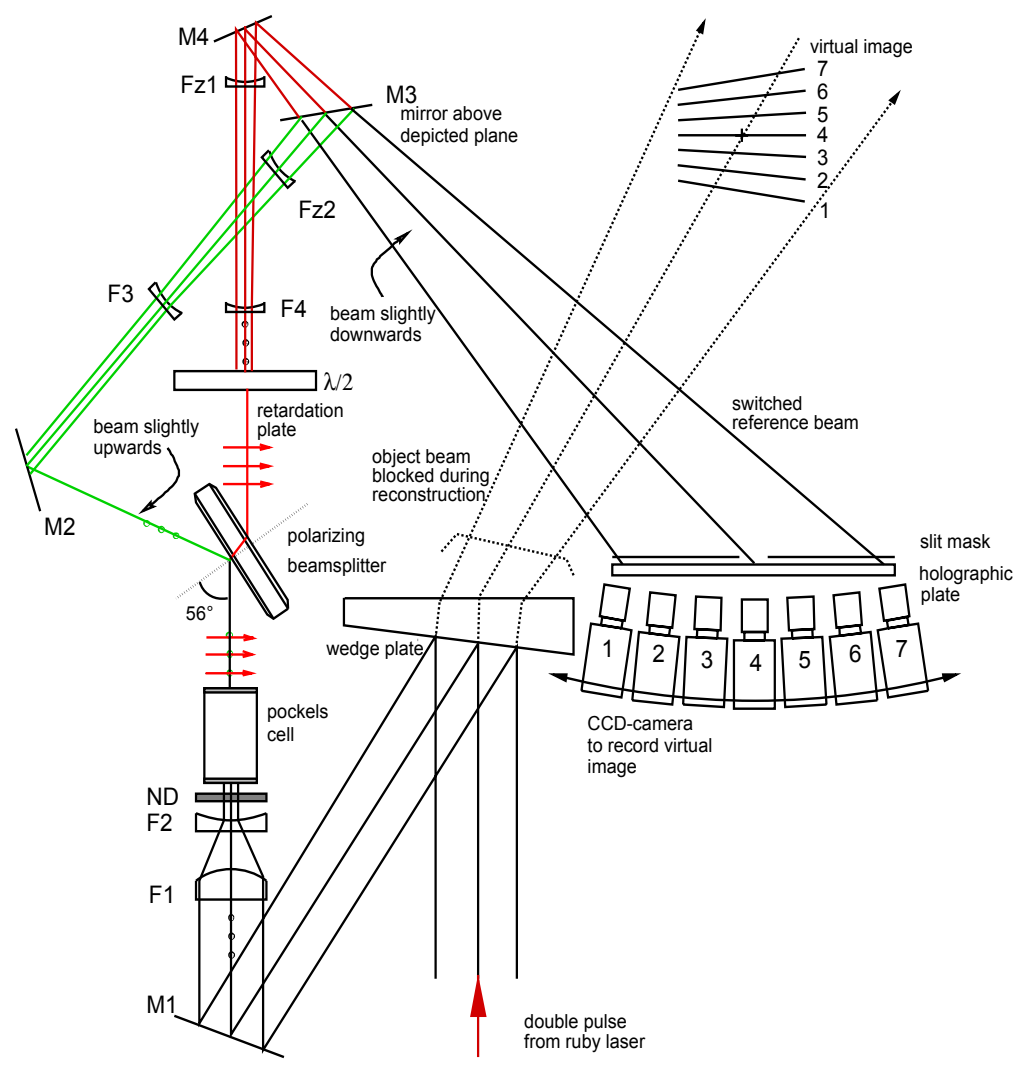

Fig. 2. Light-in-flight setup for recording with polarization-switched reference beams offering different angles of incidence. The reference beam expanded by lens F3 projects out of the paper plane to fall onto the holographic plate from above. Sheet-wise reconstruction is done with the same setup after blocking the object beam and using the reference beams one after the other. In-focus planes in the virtual image correspond to the horizontal positions of the CCD camera. 
Consequently, the first pulse is reflected while the second is transmitted at the beam-splitter. To maintain good interference on the holographic plate with the object light the polarization of the second pulse is turned back by a half-wave-plate. During reconstruction a slit mask - or simply the limiting aperture of the imaging system - is used to select the aperture in both holograms which reconstructs the same region in depth. Due to the thickness of each reconstructed shell (that depends on the coherence length of the recording light source) a joint focal plane can be imaged by the $\mathrm{CCD}$ and read out successively using each reference beam. Thus, a pair of particle field images can be obtained and processed by means of common PIV algorithms. The exact spatial orientation and depth position of each of the $2 \mathrm{D} 2 \mathrm{C}$ velocity maps is obtained from a reference hologram of a regular planar grid placed under a tilted angle, thus all maps can be rearranged into a single 3D2C map. First results have been obtained in a free jet in air during measurements in the region of transition to turbulence (Geiger et al. 2000). Up to 21 planes were obtained from which the inner 18 were used to evaluate 64.800 velocity vectors. It was found that the validity rate $(\mathrm{SNR}>1,2)$ is as good as $97 \%$. As an example, a single plane of velocity vectors with no filtering or interpolation is shown in figure 3 .

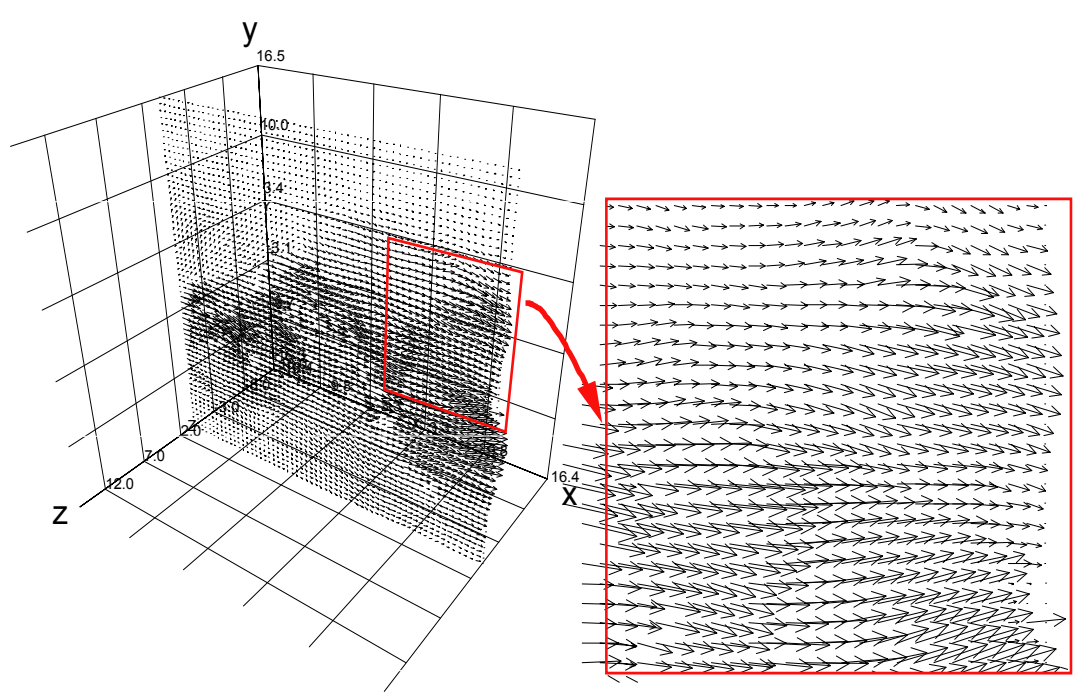

Fig. 3. Free air jet transition to turbulence. Sample plane from a LiFH-PIV measurement. The whole volume as indicated by the grid was sampled with a total of 21 such planes. 
It has been shown that cross-correlation analysis of noise-reduced holographic particle image fields from a ruby laser yields high quality measurements of even non-stationary and turbulent flows. The maximum flow velocity, however, is restricted by the minimum pulse-delay of the ruby laser (typically $\Delta \mathrm{t}=50 \mu \mathrm{s}$ ), and a cumbersome alignment procedure of the imaging system for the virtual-image readout is needed. Furthermore, only two components of each velocity vector are obtained, even though holography is capable of recording the full three-dimensional information with high spatial resolution. Interrogation of the virtual image requires a minimum working distance of the camera, which is slightly larger than the distance between holographic plate and flow (usually several ten centimeters) and thus imaging does not make use of the full resolution determined by the effective hologram-aperture used for the reconstruction. To overcome these drawbacks a dual-laser-head system was developed which allows arbitrarily short pulse separations as they are common in conventional PIV applications. Furthermore, the advantages of real-image evaluation were employed.

\section{Real-image evaluation}

It is an established fact (Vikram 1990) that the reconstruction of the real image should be preferred in particle analysis holography. Among other advantages, a real particle image - as a one-to-one copy of the particle distribution in the flow - can be scanned directly by means of a traversing CCD sensor and particle positions can be extracted immediately within a cartesian coordinate-system. Furthermore, a magnification by microscopic lenses allows even better resolved particle images since the working distance can now be kept small, but increases the amount of image data to quantities which are yet difficult to handle. Nevertheless, still nowadays the reconstruction of faithful images is a challenging task because an exact replica of a phase conjugate reference wave is a prerequisite. Improper conjugated reference beams produce considerable aberrations in the delicate particle images and displacement measurements suffer from bias errors (Chan et al. 2000; Sholes and Farell 2000).

For a high-fidelity reconstructing conjugate wave the wavelength needs to be the same as for the recording, whereas direction and curvature of the wave have to be inverted. Even slight deviations of the divergence of the order of microradians are responsible for severe aberrations. The easiest approach to achieve these requirements is the use of a horizontally incident plane reference wave and a fixed holder for the holographic plate. After 
development, the hologram is replaced in the plate holder and rotated about its vertical axis by $180^{\circ}$. Thus the plane wave used for the recording serves now for the conjugate wave in the reconstruction.

For the detailed testing of some real-image evaluation techniques a setup was developed to simulate pulsed particle holography by using a continuous wave laser. A model particle field of $10 \mu \mathrm{m}$ PMMA particles in a perspex block is illuminated with a thick light-sheet $(4-8 \mathrm{~mm})$ to provide conditions as in LiFH. The light scattered at $90^{\circ}$ is recorded twice on a holographic plate with each one of two reference beams. Between recordings, the block can be traversed or rotated for a particle displacement. A scanning CCD is then traversed through the real images which are reconstructed by illuminating the developed hologram alternately with either of the reference beams.
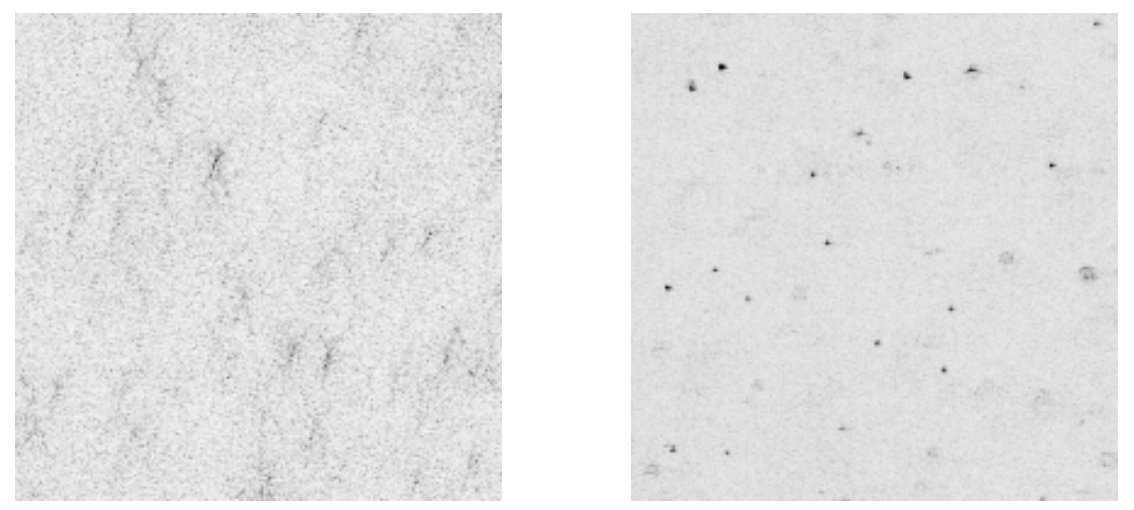

Fig. 4. Negative images of a single plane digitized from the reconstructed real images of PMMA particles in Perspex. Left: Spherical aberration and astigmatism due to an insufficiently collimated reference beam; the noise level (background speckle pattern) is rather high. Right: Nearly perfect collimation, high quality focused particle images can be observed as well as slightly out-of-focus images from adjacent planes that show up as less bright disc-like structures. Field of view: $1,8 \times 1,8 \mathrm{~mm}^{2}$.

Figure 4 shows reconstructed images to illustrate the optical requirements. The left image was obtained by adjusting low quality collimating optics by conventional methods, i.e., by checking for constant beam diameter at several distances from the collimating lens. The right image shows the result of a similar recording situation, however using a corrected lens and a nearly perfect planar wave as controlled by a shearing-plate interferometer. Comparing both images, it is obvious that an insufficient reconstruction wave causes particle image intensities to be smeared out which thus contribute to a much higher background noise, resulting in a 
drastically decreased SNR. It is thus of essential importance to use an exact reference wave already in the recording. While a true diverging or converging reference beam could be compensated during reconstruction, lens aberrations become critical when the full lens apertures have to be used to make use of the effective hologram size.

\section{Particle holography at a wavelength of $532 \mathrm{~nm}$}

Recently, Nd:YAG lasers have become popular in flow investigations since advances in solid-state laser engineering have made these systems powerful, versatile and reliable. For most applications the frequencydoubled fundamental line at $532 \mathrm{~nm}$ is used and both pulsed and continuous-mode lasers are available at this wavelength. Unlike with pulsed ruby lasers - which have been quite common in holography, whose wavelength, however is difficult to match by a powerful continuous-wave laser - it is possible to use continuous-wave Nd-YAG lasers at precisely the same wavelength for the reconstruction of holographic images recorded with a pulsed light source.

When a Gaussian or top-hat profile of the beams is needed for proper illumination, the only drawback of Nd:YAG lasers is their lower pulse energy. Typically, at a repetition rate of $10 \mathrm{~Hz}$, up to $400 \mathrm{~mJ}$ per pulse can be supplied with a Gaussian beam profile and up to $1500 \mathrm{~mJ}$ per pulse with top-hat profiles - in both cases using appropriate amplifier stages. Ruby lasers, on the other hand, provide up to about $5000 \mathrm{~mJ}$ per pulse, yet at a rate of only one double pulse per minute. This situation must be considered when designing systems for particle holography in air flows where scattering by the fairly small particles is very low.

Another topic of concern are the recording materials available for the green spectral range. Common materials in holographic metrology are silver-halide emulsions which have been studied extensively (Bjelkhagen 1995). Nevertheless, delays and special efforts due to chemical processing turn out to make these materials obsolete for many needs in metrology. This has caused the number of available materials to decrease drastically and rapid changes are observed concerning manufacturers. Since the earlier well-known materials of reasonable sensitivities in the green from Agfa (8E56) and Ilford (SP695T) are no longer produced we have considered two quite new materials for our further experiments. The sensitivity of the emulsions from Slavich (VRP-M) and HRT (BB520) was examined using a pulsed Nd:YAG laser (Coherent Infinity) with a stable and Gaussian-like beam profile in order to calculate the effective exposure. 
The pulse energy at $10 \mathrm{~Hz}$ repetition rate was determined with a power meter. After development of the holographic plate $\left(20^{\circ} \mathrm{C}\right.$ and appropriate developer solution) the resulting optical density was measured. From the resulting sensitivity plots it can be concluded that a reduction in the photographic speed by a factor between 2 and 10 has to be taken into account as compared to the common red-sensitive materials. Meanwhile, also HRT has stopped the production and Slavich's VRP-M is used for all LiFH-PIV measurements.

A change in wavelength influences also the scattering behavior of tracer particles which are used to seed the flow. To predict the scattering behavior at $532 \mathrm{~nm}$ for different seeding materials used in LiFH-PIV a theoretical investigation using Mie-theory (Fortran code according to Bohren and Huffman 1983) was conducted. In figure 5 the scattering diagrams for the commonly used DEHS particles of different sizes are shown. The light, polarized perpendicular to the scattering-plane, is incident from the left. The most important part in $\mathrm{LiFH}$ is the near-backscattering regime $\left(180^{\circ}-\right.$ $210^{\circ}$ ) which shows strongly modulated intensities for all particles - especially pronounced, however, for those particles commonly used to probe turbulent air flows (diameter $\mathrm{d} \leq 1 \mu \mathrm{m}$ ).

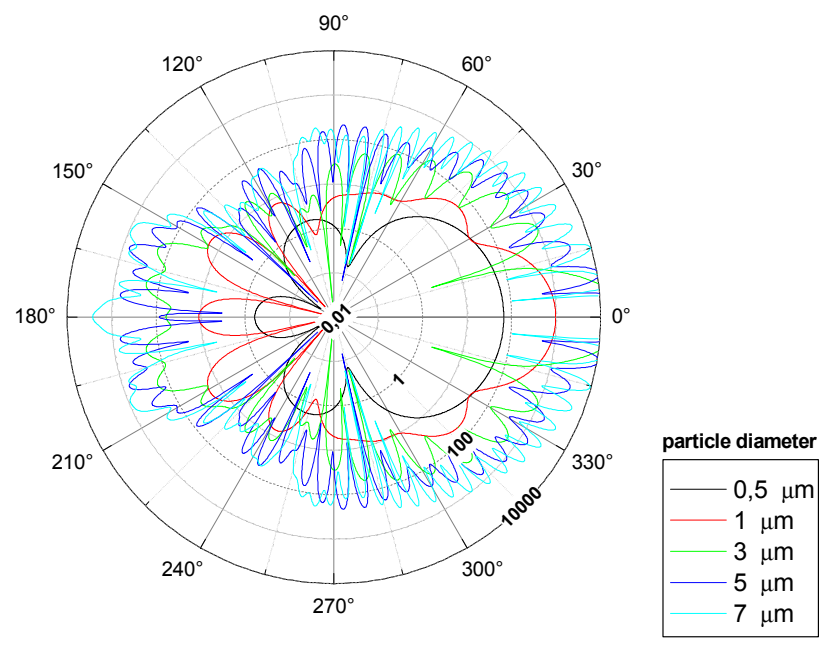

Fig. 5. Mie-scattering diagram for different sized DEHS particles in air with refractive index $\mathrm{n}=1,4527$ illuminated at a wavelength of $\lambda=532 \mathrm{~nm}$.

The scattering spectrum is recorded only partly by the hologram due to its finite size and limited dynamical range which results in a variety of spu- 
rious effects in the reconstructed image ( $\mathrm{Pu}$ et al. 2002). For LiFH-PIV, however there are no detailed experimental results yet to quantify such influences. Yet, in our experiments on the model particle field we observed varying particle image intensities and shapes when reconstructing from different apertures. The previous considerations were basis for system decisions to realize a LiFH-PIV working at $\lambda=532 \mathrm{~nm}$ and employing the advantageous real- image evaluation. The Nd:YAG laser-system, described in the following was designed in close contact with the manufacturer and offers a couple of features of great importance for particle holography. In its present configuration it is unique world-wide and can be compared only to a similar system operated at the German Aerospace Center (DLR) at Göttingen.

With reference to figure 6, both component lasers each consist of two instable Gaussian resonators (two Nd:YAG rods each, Q-switched operation, seeded by a common diode-pumped cw-Nd:YAG laser) followed by two amplifier stages and a second-harmonic generator (SHG) for frequency doubling. To ensure flexibility of the system the user can choose between different output ports and between vertical or horizontal polarization for each beam.

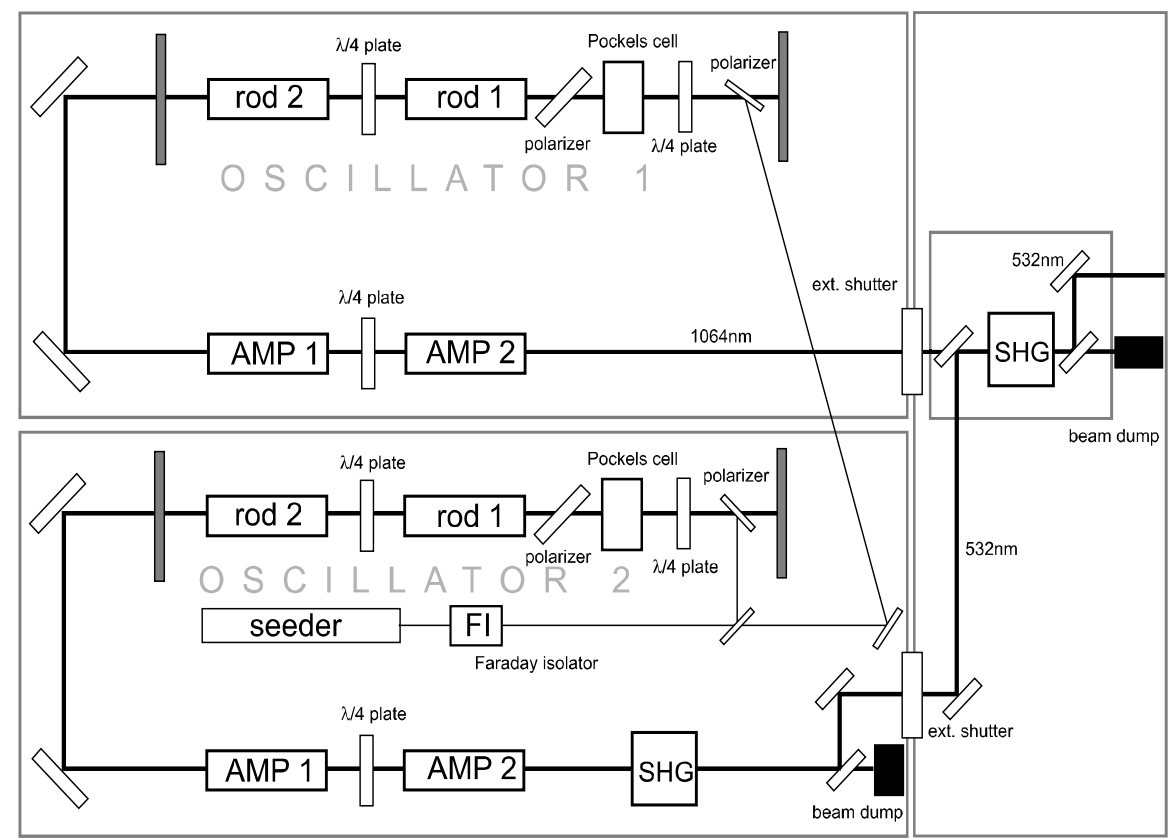

Fig. 6. Sketch of the principal layout of the double-head laser system with second harmonic generation $(\mathrm{SHG})$ and seeder. 
The two beams can either be superimposed before or after the external SHG-unit. The flashlamps and Pockels cells are driven at $10 \mathrm{~Hz}$ repetition rate resulting in maximum pulse energy of 1,6 $\mathrm{J}$ (specified 1,2 $\mathrm{J}$ per pulse). To extract a double pulse with variable time separation two external shutters accepting TTL signals can be used. Thus, constant beam properties are guaranteed which requires a constantly running system (heat-up times approximately $20 \mathrm{~min})$. The seeder $(20 \mathrm{~mW}$, cw Nd:YAG at $\lambda=1064 \mathrm{~nm})$ can be switched on or off, such that the coherence properties can be chosen to suit LiFH or conventional holography. Furthermore, it serves both resonators and ensures mutual coherence between the two output beams. The system is further equipped with additional options to provide for doublepulsing of each cavity within one pumping-cycle of the flashlamps which renders the possibility to produce up to four pulses. In addition, quite stable beam profiles are obtained over the entire lifetime of the flashlamps by adapting their driving voltage with increasing age. An improved beampointing stability is achieved by an additional feedback control system using a far-field detector and a corrective mirror.

In the configuration used for the experiments described below, the beams are cross-polarized and overlapped before entering the external SHG. Thus, once again, the reference beams are separable by a polarizing beamsplitter, while only a single optical path must be provided for the object beam.

\section{Cross-correlation LiFH-PIV for wind-tunnel applications}

A revised LiFH-PIV setup with the new laser-system - suitable for windtunnel measurements - was developed and tested in Oldenburg. To demonstrate its feasibility a wind-tunnel measurement was then conducted at DLR Goettingen using the available local laser-system that is comparable in performance. For the analysis of the holograms the reference beam geometry used in Goettingen was replicated in a separate setup at Oldenburg equipped with a fully automatic read-out unit controlled by a PC. Furthermore, a method was used that allows to extract particle images even from holograms that suffer from low diffraction efficiency due to the small scattering signals from tiny tracer particles (Herrmann and Hinsch 2001). This technique employs particle light integration by long-exposure recording during read-out and is only limited by background noise from the photographic emulsion and other scattering sources. 


\section{Recording setup}

In figure 7 the optical setup at the wind tunnel is shown true to scale. The open test section is about two meters wide and one meter deep. In the flow a generic airfoil produced two counter-rotating tip-vortices. A reference signal is produced by the first reflection from a glass wedge plate that is split into two polarization dependent reference beams by a polarizing beam splitter (TFP). A detailed side view showing both arms above and underneath the tunnel outlet is given in figure 8. A prism was mounted on a mechanical translation stage to control the path length of each reference beam. Since the coherence length of the unseeded laser is some $7 \mathrm{~mm}$, the path lengths need to be identical within $1 \mathrm{~mm}$ compared to the path length of the object beam. This travels over three bending mirrors (HEM) before it is expanded by a set of two coated lenses to illuminate a small part of the wake flow behind the profile.

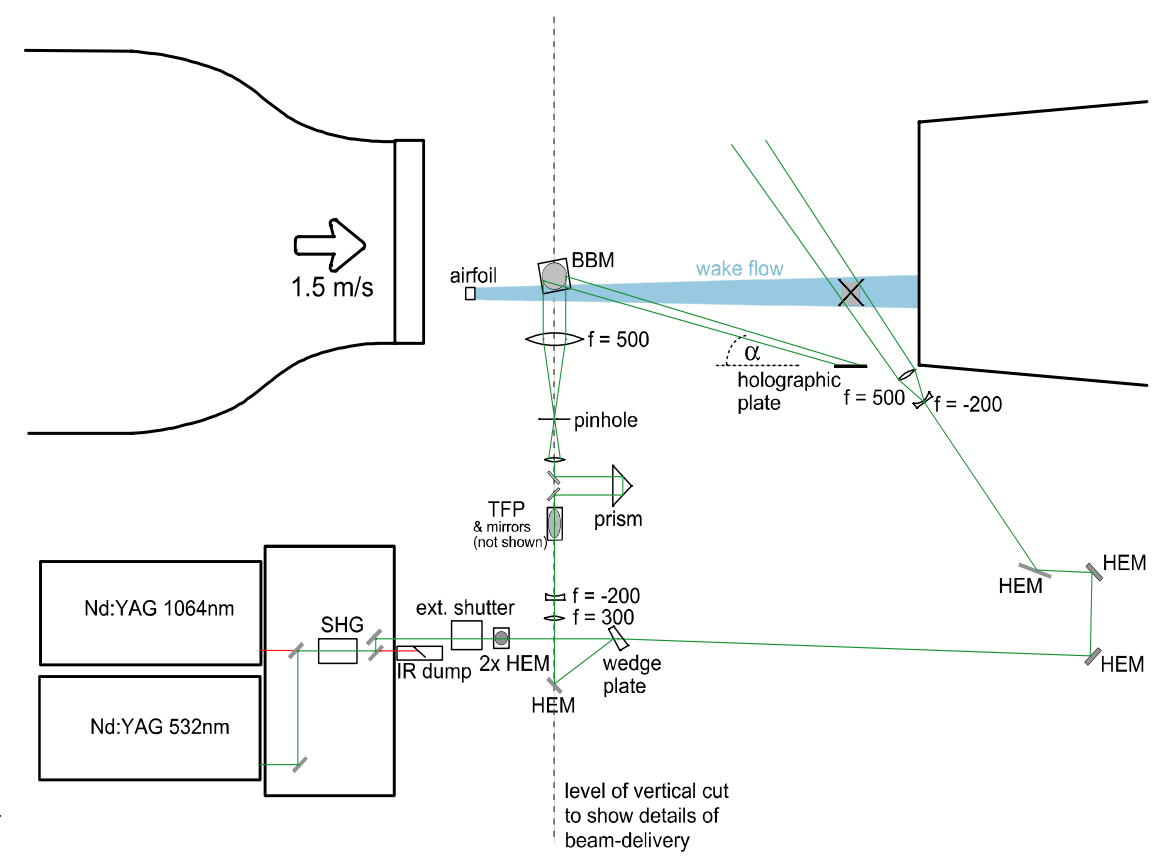

Fig. 7. Setup for recording LiFH-PIV holograms at the $1 \mathrm{~m}$ wind tunnel at DLR in Göttingen, showing the beam paths from the laser heads through the combining optics and shutter, two mirrors (HEM) for adaption of the beam height to fit the measurement area and the optics for holography as explained in the text. For the out-of-plane propagation and details of the reference beams as indicated by the dashed line see Fig. 8. 
The position of the center of the measurement volume is located at a distance of $34,5 \mathrm{~cm}$ from the holographic plate, which was placed exactly at the flow boundary - far enough to avoid vibrations affecting the stability of the measurement system, but close enough to ensure high signals from the scattering particles. The delivery of the reference beams was realized in a vertical arrangement in order to maintain the same azimuthal angle $\alpha$ of incidence onto the holographic plate (cf. the earlier explanations concerning light-in-flight holography), while the two recordings are distinguished by their height-angles $\pm \beta$. Perfect collimation of the reference beams was controlled by the shearing interferometer. The beam profiles of the two laser heads showed intensity variations typical of a multi-mode laser which are changing with increasing distance from the laser-head aperture. Since a low pulse energy in the reference beams is already sufficient for a proper plate exposure, pinholes could be introduced without damages to improve the beam profile.

A couple of holograms have been recorded under different conditions regarding type and size of particles as well as density - all having in common a very low amount of object light as compared to the reference light intensities. The mean flow of about $1,5 \mathrm{~m} / \mathrm{s}$ produced rather stable vortices, but with fluctuating positions. Since there was no control to trigger the events, it turned out to be difficult to catch single vortices by the holographic method.

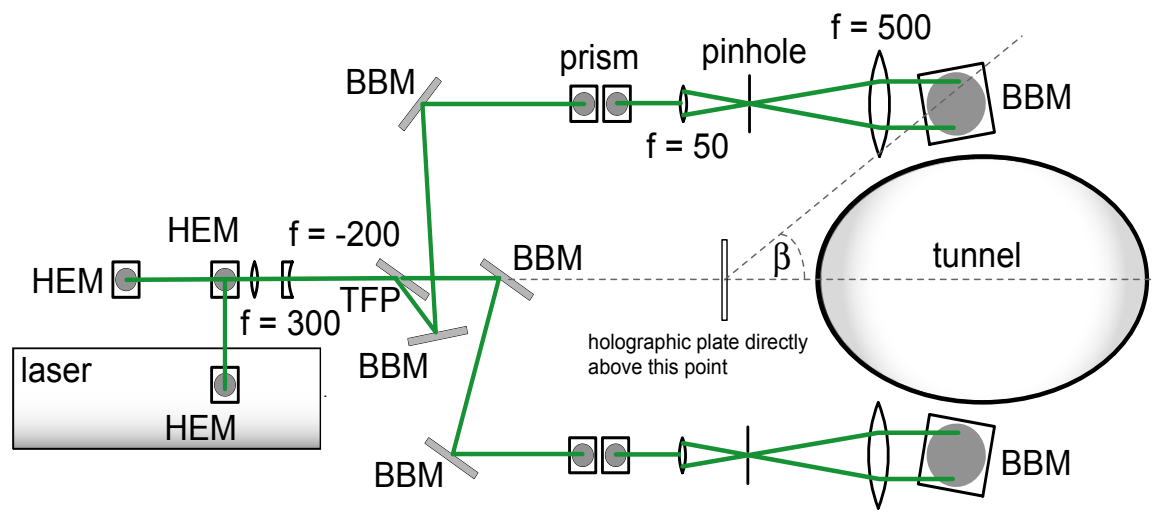

Fig. 8. Side view of the reference beams with spatial filters (pinhole) for a more uniform illumination of the holographic plate. Given in gray are the outlet of the wind tunnel and the cover box of the combining optics. The thin-film polarizer (TFP) directs each pulse according to its polarization into one of the optical arms, guided by specially coated broad-band-mirrors (BBM). The beam is then directed out of the projected plane towards the holographic plate. 


\section{Reconstruction setup}

To reconstruct particle image fields from the wind-tunnel-flow holograms - recorded under LiFH conditions - and extract image data throughout the whole volume an automatic read-out setup was developed. It comprises of the optical setup, two shutters, two mechanical translation stages and a controlling and image-acquisition unit based on a single PC with large storage capacities, a frame-grabber and a CCD camera module. In the following some details of this setup are described.

For this off-site reconstruction a continuous wave laser (Nd:YAG at $\lambda=532 \mathrm{~nm}$ with $150 \mathrm{~mW}$ in single longitudinal mode) with Gaussian beam profile is used. A top view of the beam handling unit (lower part of the setup) is shown in figure 9.

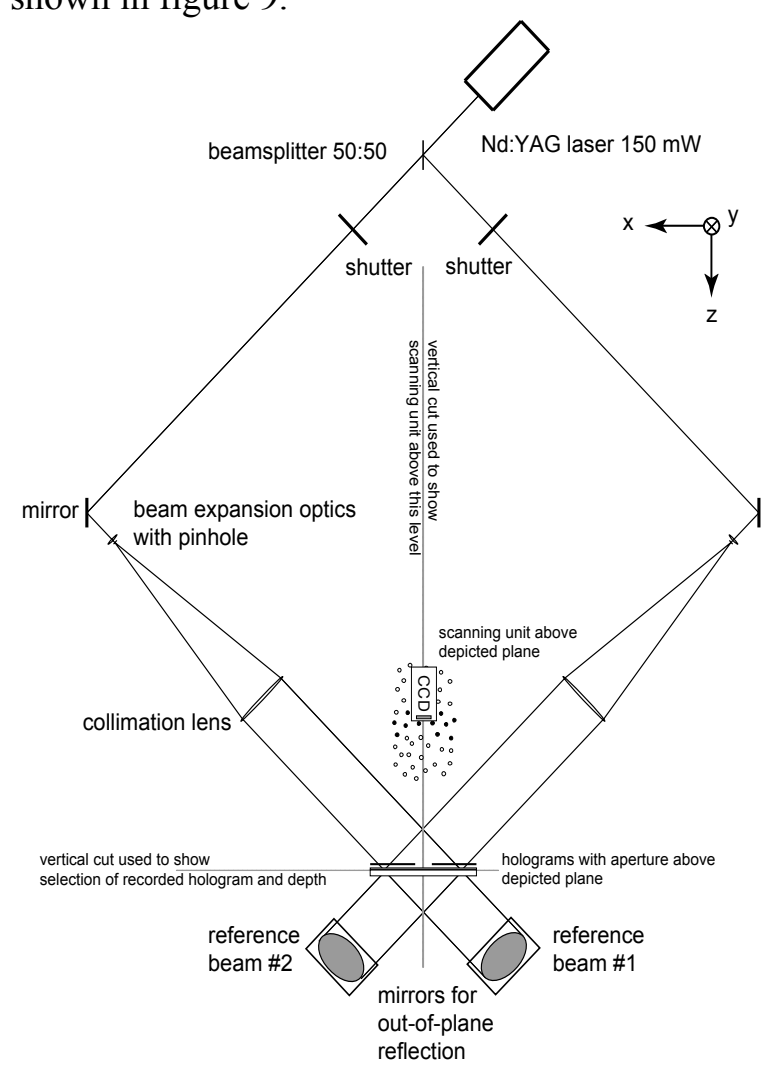

Fig. 9. Beam handling unit of the reconstruction setup for extracting real particle image fields from wind-tunnel recordings. Carefully collimated beams are propagating out of the plane to illuminate the holograms placed above, while the reconstructed real-image can be scanned by a CCD sensor. 
Both the reference beams are expanded, collimated and directed out of the plane by two highly planar $(<\lambda / 20)$ mirrors to illuminate the holograms. The collimation is checked by shearing plate interferometer. The relative positions of hologram and mirrors are precisely scaled down from the arrangement at the wind tunnel to produce the same angles of incidence as before. Above this beam-handling unit, the scanning unit is placed next to the holographic plate (figure 10 left, showing a vertical cut as indicated in figure 9). For the sake of convenience, the complete arrangement has been turned by $90^{\circ}$ with respect to the original recording orientation and the illumination of the plate (figure 10, right) is from below. Thus, angle $\beta$ is now the originally vertical component of the angle of incidence while the originally azimuthal component $\alpha$ determines the outof-plane propagation direction in figure 9. Both the mechanical shutters, controlled by the computer, are used to block either reference beam.

Figure 10 indicates also the areas exposed by the two recording reference waves in gray shade, i.e., the contours of the holograms. They are elliptical because of the oblique incidence of the circular beams and they do not coincide because of their different height-angles $\pm \beta$. The exact alignment of both beams is done by adjusting the mirrors with micro-screws under visual inspection of a calibration hologram.

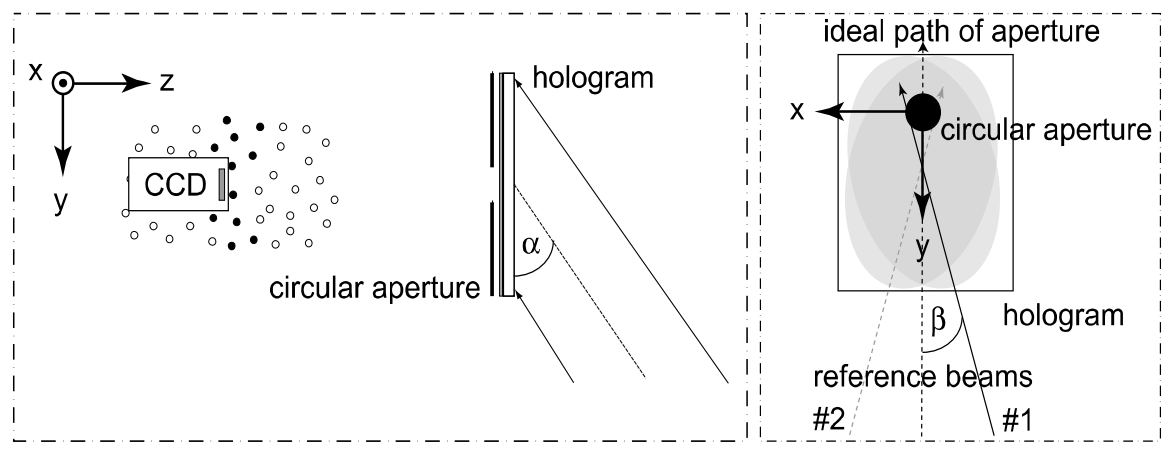

Fig. 10. Upper part of the reconstruction setup, showing the scanning unit (left) and the beam propagation towards the holograms (right). A moving aperture is used to select the corresponding depth according to the position of the sensor, while the beams are switched to reconstruct either particle field.

During reconstruction, a circular aperture is moving along the bisector of the "propagation axes" (the tracks of the plane of incidence of the reference beams on the hologram) to select the region of depth currently under investigation. This aperture can be moved by a translation stage along the $\mathrm{x}$ - and $\mathrm{y}$-direction. Another translation stage - in this case three- 
dimensional - controls the camera position as shown in the left part of figure 10. Both translation stages as well as the image acquisition and the exposure times of the CCD sensor are controlled by the PC.

At each position of the sensor the reference beams are used alternately (switched by the mechanical shutters) to acquire both the successive images without moving the sensor. This avoids that a repositioning error can enter into the displacement measurement. A typical scanning process is started for a given xy-coordinate of the CCD-sensor. The sensor is then scanned along the z-direction while the position of the circular aperture on the hologram (setting the depth region) is following to provide reconstruction of the corresponding depth position. After a scan over the full depth is finished the same procedure is repeated for another xy-coordinate. Providing perfect alignment, a single aperture position should allow reconstruction of the same depth slice for both reference beams. Unfortunately, we have not yet succeeded in achieving the ideally aligned setup. This became evident when comparing both the reconstructed slices. Thus, a relationship between aperture position and reconstructed volume element was established empirically for each hologram to control aperture positions and sensor positions throughout the whole scanning process.

It was already mentioned that a long-time exposure of the CCD sensor is used to compensate for low diffraction efficiencies of the particle holograms (Herrmann and Hinsch 2001). This procedure was necessary for all evaluations of the flow holograms from the DLR wind tunnel experiments as exposure times usually used in CCD sensors (typically $\tau=100 \mu \mathrm{s}$ ) turned out to be insufficient for proper image quality. By carefully minimizing all scattered light reaching the sensor from directions other than the hologram it was indeed possible to extract long-exposure (up to $\tau=2 \mathrm{~s}$ ) particle images with a good signal-to-noise ratio. Scanning times, of course, have been increased drastically - yet it becomes possible to record volumes of sufficiently larger cross-sectional size.

The digital images are stored on a hard-disc drive, each identified by its position in space and the according reference beam. For their evaluation a three-dimensional gray-value correlation is performed. A first version of a data handling routine has been implemented in Matlab ${ }^{\circledR}$, assembling subvolumes from the image data and carrying out the correlation by threedimensional FFTs. The demand on memory and computing power is still enormous and should be relaxed by further refinements. The present procedure represents a straightforward solution to provide input data for extended common PIV algorithms. It should be mentioned that the current version is still a very simple implementation examining the correlation results by means of their maximum values and applying sub-pixel algorithms 
to extract more accurate displacement data. Window-shifting techniques or validation criteria have not yet been implemented.

\section{Results on wind tunnel flow}

All holograms recorded at the wind tunnel have been examined in a first step as to their particle image quality and density. In an intermediate check during the campaign at DLR it turned out, that appropriate particle image densities are only obtained by very long operating times of the seedinggenerator. To obtain a sufficient number of larger particles the generator was operated with lower pressure settings - that achieve a broader particle size distribution than commonly used in wind tunnel measurements - for up to one hour. According to previous measurements of size distributions about $50 \%$ of the particles were smaller than $1 \mu \mathrm{m}$ and only $7.5 \%$ reached sizes larger than $3 \mu \mathrm{m}$.

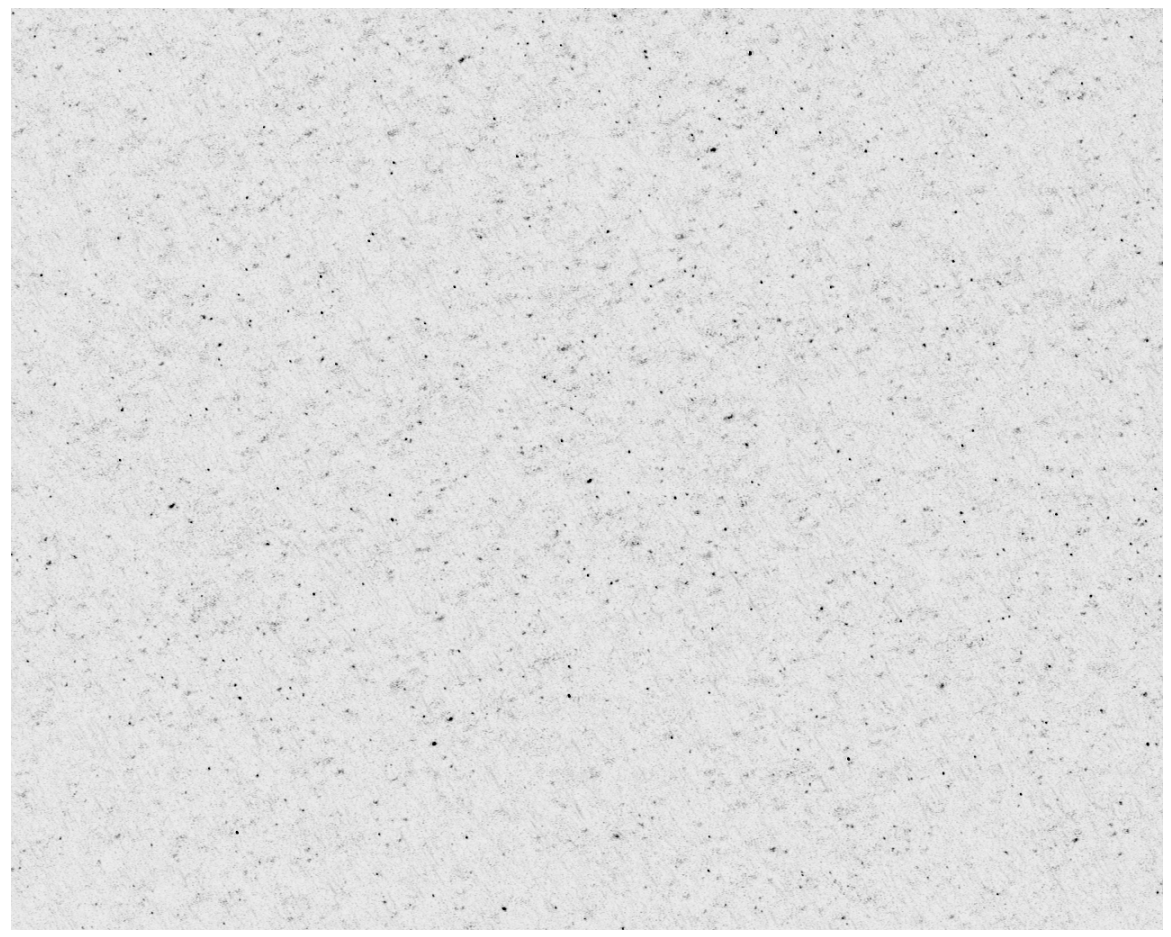

Fig. 11. Inverted image of a digitized plane $(1280 \mathrm{px} \times 1024 \mathrm{px})$ from a reconstructed real image of a wind tunnel flow (DEHS particles after one hour of operation of the seeding generator). The image was obtained by an exposure time of 0,5 seconds, the field of view is $8,6 \times 6,9 \mathrm{~mm}^{2}$. 
With a cross-sectional dimension of the object light beam of about $4 \mathrm{~cm}$ the scattered light intensities have thus been rather low. Judging from the type of reconstructed images we assume that only larger particles contribute to the signals obtained from long-exposure reconstructions. Unfortunately, in most cases the particle image densities have still been too low to allow for a proper correlation analysis on the images obtained.

For the best hologram the particle density is high enough to evaluate the complete set of images by three-dimensional cross-correlations on $128^{3} \mathrm{px}$-sub-volumes. A sample plane from the reconstructed image is shown in figure 11. Thus, a volume of $24,0 \times 18,8 \times 29,1 \mathrm{~mm}^{3}$ was scanned within 35 hours of operation time of the read-out-unit, the bottleneck still being the slow translation stage moving the circular aperture over the hologram between exposures.

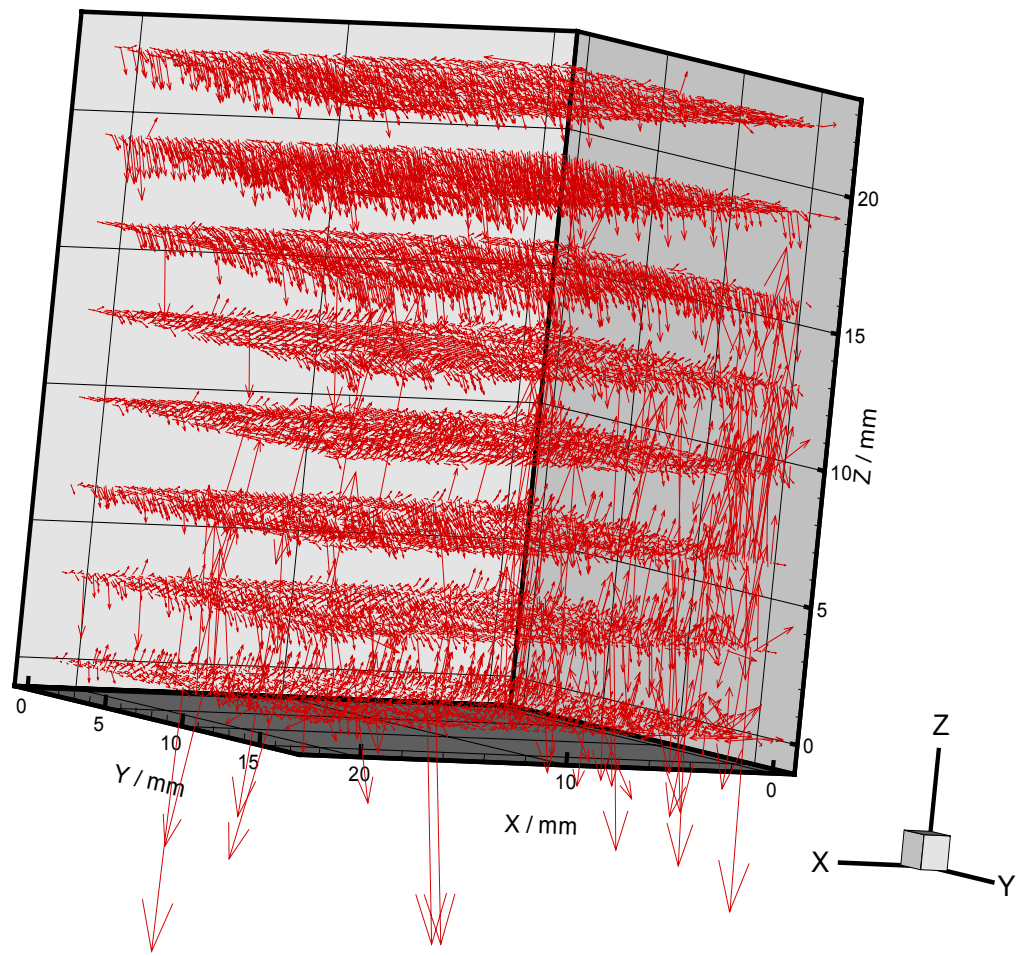

Fig. 12. Evaluated wind tunnel flow, 16.640 vectors have been obtained by threedimensional gray-value correlation. The plane-like distribution of the vectors is a result of a relatively large separation between adjacent image slices, from which 128 enter in each correlation. 
The result are 5409 image pairs, each 2,5 MB in size - in total more than $13 \mathrm{~GB}$ of image data. Despite the maximum depth resolution $(9,8 \mu \mathrm{m})$ of the translation stage, adjacent image planes have been acquired with a separation of $49 \mu \mathrm{m}$ to facilitate the use of sub-pixel algorithms and to prevent from over-sampling the elongated particle images. Thus, the resulting interrogation volumes are $857 \times 857 \times 6272 \mu \mathrm{m}^{3}$ in size.

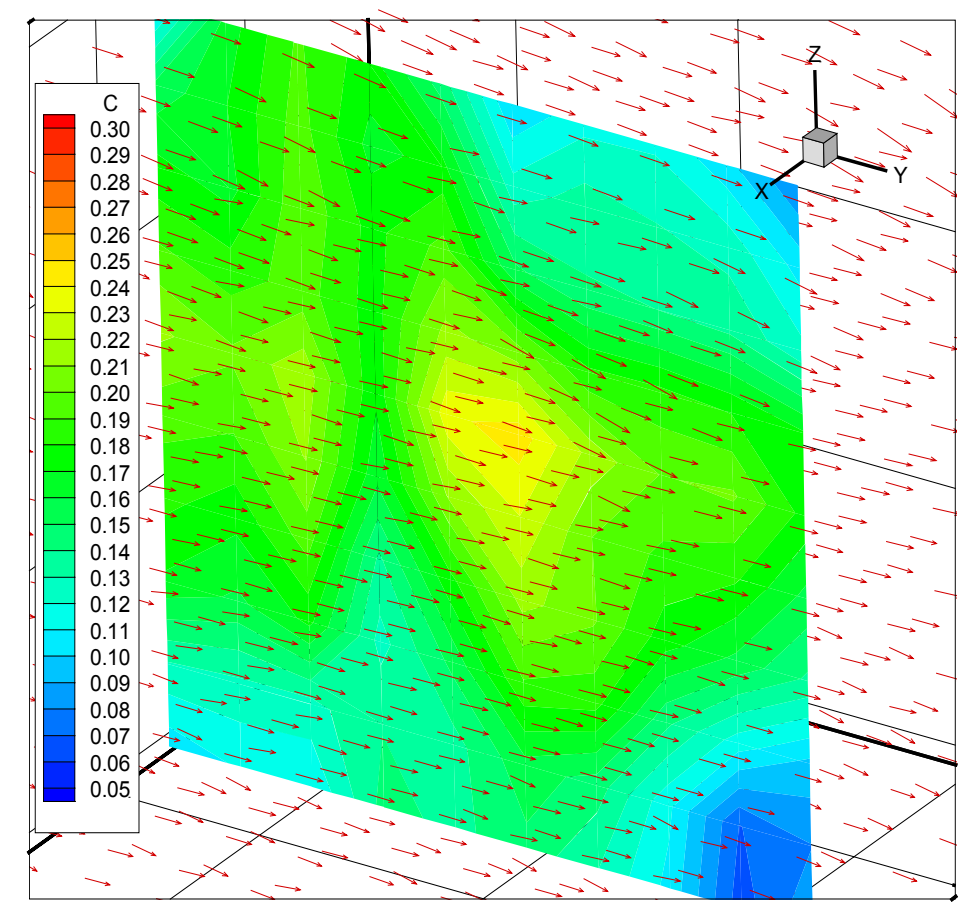

Fig. 13. Detailed view, from inner part of the evaluated volume, showing the three-dimensional velocity distribution and the correlation-coefficient (C) in one slice as a measure for the reliability of the velocity value.

As mentioned before, the moving vortices behind the generic air-foil did provide an unfavorable flow configuration. A single snap-shot hologram of a small region is unlikely to reveal characteristic parts of a single vortex. Therefore, it is not astonishing that the hologram yields mainly the mean velocity of the wind-tunnel flow. Figure 12 shows the complete correlation result, a total of 16.640 vectors have been obtained from an evaluation with $50 \%$ overlap of the interrogation cells (IC) in each direction. The plane-like distribution is a result of the densely-spaced grid points along 
the $x$ - and $y$-direction and the much wider spacing (approx. $\times 7,3$ ) along the $\mathrm{z}$-direction. Beside some spurious vectors a net flow perpendicular to the mean flow is observed in some regions.

A detailed view of the velocities in the interior of the flow field is shown with their corresponding correlation coefficient in figure 13. Here, an overlap of $75 \%$ was used, increasing the total number of vectors to 124.800 within the same volume. Even if these results do not furnish important flow data, it was shown that particle images could be extracted, of a quality sufficient for further processing - yet with still too low a particle density. A maximum depth of $47 \mathrm{~mm}$ has been covered from other holograms, the useable cross-section of the measurement volume extended to $30 \times 30 \mathrm{~mm}^{2}$ providing particles of sufficient brightness even at its border. Increasing seeding densities could allow for smaller ICs, with 64px or even 32 px side-length and also non cubic ICs are feasible, but have not yet been implemented in the basic code.

\section{Conclusions}

It has been shown, that the use of a high-power Nd:YAG laser system allows to measure three-dimensional velocity distributions with reasonable resolution in a large wind-tunnel environment. This was achieved by lightin-flight holography (LiFH-PIV), a method based on a holographic tomography technique which provides high-quality particle images even in deep volumes. The technique is capable of effectively reducing the limiting noise level in such images, which is determined by the number of unfocused background tracer particles - thus limiting the maximum depth of the measurement volume.

Furthermore, a principle to reconstruct weak holographic particle images at the noise limit has been successfully applied to holograms with low diffraction efficiency. It allowed to use small tracer particles in a fully seeded open test-section. However, despite the powerful laser-system mostly only the larger particles around $3 \mu \mathrm{m}$ in size were recorded. Thus, it is still a challenging task to reconstruct particles of around $1 \mu \mathrm{m}$ in size in densely seeded volumes. More experimental investigations on the influence of scattering by these particles are needed. For the long-time exposure then to work all further noise contributions have to be carefully minimized to obtain a good SNR.

The effort to set up and calibrate the recording and reconstruction optics is still large. This is mainly due to the optical layout in three dimensions necessary to avoid aperture switching between the recording beams in 
LiFH. Since problems have been encountered with this scheme anyway, alternative layouts deserve consideration that resume two-dimensionality. It still is a great advantage that the independent evaluation at an extra site does no longer block the flow facility.

\section{References}

Abramson N (1996) Light-in-flight or the holodiagram: the columbi egg of optics. SPIE Optical Engineering Press, Bellingham, Wash

Barnhart DH, Adrian RJ Papen GC (1994) Phase-conjugate holographic system for high-resolution particle image velocimetry. Appl Opt 33:7159-7170

Bjelkhagen HI (1995) Silver-halide recording materials for holography and their processing. Springer-Verlag, Berlin

Bohren CF and Huffman DR (1983) Absorption and scattering of light by small particles. Wiley Science, New York

Chan KT, So RMC, Wong WO, Li YJ (2000) Particle image aberrations in offaxis holography. In: Carlomagno GM, Grant I (eds) $9^{\text {th }}$ Int. Symp. on Flow Visualization, Edinburgh

Geiger M, Herrmann SF, Hinsch KD, Peinke J (2000) Analysis of free jet turbulence with cross-correlation light-in-flight holography (LiFH). Proc. Euromech 411, Rouen

Herrmann SF, Hinsch KD (2001) Particle holography and the noise limit. In: Kompenhans J (ed) 4th Int Symp on Particle Image Velocimetry, DLRMitteilung 2001-03, Göttingen

Hinrichs H, Hinsch KD, Kickstein J, Böhmer M (1997) Light-in-flight holography for visualization and velocimetry in three-dimensional flows. Opt Lett 22:828830

Hinrichs H, Hinsch KD, Netter R, Surmann C (1998) Light-in-flight particle holography for velocimetry in a wind tunnel. In: Carlomagno GM, Grant I (eds) $8^{\text {th }}$ Int. Symp. on Flow Visualization, Sorronto, pp 19.1-19.5

Hinsch KD (2002) Holographic particle image velocimetry. review article Meas Sci Technol 13:R61-R72

$\mathrm{Pu}$ Y, Cao L, Meng H (2002) Fundamental issues and latest development in holographic particle image velocimetry. International Mechanical Engineering Congress \& Exhibition, Paper 33171, ASME, New Orleans

Sholes K, Farrell PV (2000) Optical alignment-induced errors in holographic particle image velocimetry. Appl Opt vol 39 31:5685-5693

Tao B, Katz J, Meneveau C (2000) Geometry and scale relationships in high Reynolds number turbulence determined from three-dimensional holographic velocimetry. Physics Of Fluids Lett, vol 12, 5:941-944

Vikram CS (1990) Holographic particle diagnostics. SPIE Milestone Ser MS21, Bellingham, Wash

Zhang J. Tao B, Katz J (1997) Turbulent flow measurement in a square duct with hybride holographic PIV. Exp Fluids 23:373-381 